\title{
Treatment of avoidant/restrictive food intake disorder: a systematic review
}

\author{
Anna Dalle Grave \& Walter Sapuppo \\ Sigmund Freud University. Ripa di Porta Ticinese, 77, 20143 Milano, Italy.
}

\section{Keywords}

Avoidant/restrictive food intake disorder Treatment

Cognitive behavior therapy

Family-based treatment

Eating and feeding disorders

\begin{abstract}
Objective: This study aimed to conduct a systematic review on the effects of available treatments for avoidant/restrictive food intake disorder (ARFID).

Methods: Literature searches, study selection, method development, and quality appraisal were performed independently by one author, and data were synthesized using a narrative approach. Studies published in English and in peer-reviewed journals that evaluated the outcomes of ARFID treatments in at least three participants were taken into consideration, while non-original studies, editorials and letters to the editor were excluded.
\end{abstract}

Results: The review had three main findings. First, in all of the studies by the end of the treatment there was a significant weight gain in the ARFID participants. Second, weight recovery was maintained at follow-up in a good percentage of participants with an associated improvement of eating behavior and a reduction in anxiety, depression, and fear for the adverse consequences of eating. Third, there was weak or missing evidence regarding the treatment of ARFID in adults and the difference in outcome in the three different ARFID profiles. Eighteen studies were selected and eleven were included.

Conclusion: The treatments for ARFID, although promising, have focused only on young participants and addressed the normalization of eating with behavioral procedures, but not the underlying psychopathology. Moreover, the lack of randomized controlled trials with adequate statistical power does not allow to draw conclusions on the difference in the effectiveness of the interventions tested.

\section{Introduction}

The fifth edition of the Diagnostic and Statistical Manual of Mental Disorders (DSM-5) (American Psychiatric Association, 2013) has merged eating disorders and the disorders of early childhood or early adolescence, that were classified into two separate diagnostic categories in the DSM-IV (American Psychiatric Association, 2006), in the single diagnostic category "feeding and eating disorders".
Among this, avoidant/restrictive food intake disorder (ARFID) replaces the "childhood or early childhood eating disorder", which was a diagnosis limited to children of six years or younger in the DSM-IV (American Psychiatric Association, 2006). Currently, ARFID has no age limit and can also be diagnosed in teenagers or adults.

ARFID, according to the diagnostic criteria of DSM-5 (American Psychiatric Association, 2013), is an eating disorder that can occur as a consequence of an apparent lack

Copyright $\odot 2020$ Anna Dalle Grave. This is an open-access article distributed under the terms of the Creative Commons Attribution License (CC BY). The use, distribution or reproduction in other forums is permitted, provided the original author(s) and the copyright owner(s) are credited and that the original publication in this journal is cited, in accordance with accepted academic practice. No use, distribution or reproduction is permitted which does not comply with these terms.

Anna Dalle Grave (₫) annadallegrave@gmail.com

Received: 24 April 2020; Accepted: 29 April 2020; Published online: 15 May 2020. doi:10.32044/ijedo.2020.04 
of interest in eating or food or avoidance based on the sensory characteristics of food or a concern about the negative consequences of eating (American Psychiatric Association, 2013). The disorder is characterized by a persistent inability to meet appropriate nutritional and/or energy needs associated with one (or more) of the following factors: (i) significant weight loss (or failure to achieve expected weight gain or discontinuous growth in children); (ii) significant nutritional deficits; and (iv) marked interference with psychosocial functioning (Norris et al., 2018). The disorder is not better explained by a lack of food availability or by a culturally sanctioned practice (American Psychiatric Association, 2013) and does not present concerns about weight and body shape typically associated with anorexia nervosa (Norris, Spettigue, \& Katzman, 2016). Finally, the disorder is not attributable to a concomitant medical illness or is not better explained by another mental disorder (American Psychiatric Association, 2013).

After the introduction of ARFID as a distinct diagnostic category of eating disorders (American Psychiatric Association, 2013), numerous articles have been published that have evaluated the effects of specific therapeutic interventions for the management of this disorder, but so far no systematic review has been conducted on this important clinical issue.

The objective of this systematic review was to evaluate the treatments available for ARFID according to the PICOS formulation (P - problem / patient / population; $\mathrm{I}$ - intervention; C - comparison/control; O - outcome; S - study design) (Richardson, Wilson, Nishikawa, \& Hayward, 1995), focusing in particular on their effects on weight modification, dietary behavior, dysfunctional beliefs, anxiety levels and fear of adverse consequences.

P-Population: individuals in the general population with ARFID. I-Intervention: (i) cognitive behavioral therapy (CBT); (ii) exposure and response prevention (ERP); (iii) family-based therapy for ARFID (FBT-ARFID); (iv) dialectical behavioral therapy; (DBT); (v) integrated food aversion treatment (iEAT); (vi) pharmacological therapy; (vii) intensive treatments (residential and semi-residential). C-Comparison: (i) no control groups; (ii) healthy controls; (iii) controls in waiting list. O-Outcome: (i) weight gain; (ii) resolution of nutritional deficiencies; (iii) modest expansion of food variety; (iv) decrease in beliefs in dysfunctional cognitions and anxiety levels; (v) greater flexibility of refused foods; (vi) decrease in fear of adverse consequences. S-Study Design: (i) retrospective studies; (ii) case reports; (iii) randomized controlled trials (RCTs); (iv) longitudinal cohort studies.

\section{Experimental Section}

The review was completed following the Preferred Reporting Items for Systematic Reviews and Meta-Analyzes (PRISMA) guidelines (Liberati et al., 2009).

\section{Inclusion and Exclusion Criteria}

All studies published in English and in peer-reviewed journals that evaluated the outcomes of ARFID treatments in at least three participants were taken into consideration. Non-original studies have been excluded, including editorials and letters to the editor. The date publication range was selected from 2013 to 2019, as the criteria for ARFID were introduced in 2013 with the DSM-5.

\section{Information Source and Search Strategy}

Literature searches were performed on PubMed. The following MeSH terms were used to perform the systematic search: \# 1 Avoidant, \# 2 restrictive, \# 3 eating, \# 4 food, \# 5 intake, \# 6 food intake, \# 7 disease, \# 8 disorder, \# 9 ARFID, \# 10 feeding and eating disorders, \# 11 feeding, \# 12 disorders, \# 13 eating disorders. The following combinations were applied as search parameters: (\# 1 AND \# 2 AND \# 3 OR \# 4 AND \# 5 OR \# 6 AND \# 7 OR \# 8 OR \# 9 AND \# 10 OR \# 11 AND \# 12 OR \# 13).

\section{Study selection}

Electronic literature searches and study selection on the basis of methodology and appropriateness for inclusion were carried out independently by one author (A.D.G.). The National Institute for Health and Clinical Excellence (NICE) checklist (National Institute for Health and Clinical Excellence, n.d.) was used for quality appraisal in non-controlled studies $(n=9)$. A score of $0-3$ was considered to be of low quality; between $4-6$, moderate quality; and $>7$ high quality (Table 1). For controlled studies $(n=2)$, the quality appraisal was carried out according to the Newcastle-Ottawa (NOS) scale (Stang, 2010) which is based on a 9-star system in which the scores of 0-3 were considered low quality, 4-6 moderate quality and 7-9 high quality (Table 2).

\section{Data Collection Process}

As a first step, the title and abstract of each article were screened for language and relevance of subject matter. In the next phase, studies were assessed to determine methodological quality and appropriateness for inclusion. The details of the selected studies are presented in Table 3, which reports the authors, the year of publication, the type of study, the sample, the control, the intervention, and the main findings. 
Table 1. Quality appraisal for non-controlled studies

\begin{tabular}{|c|c|c|c|c|c|c|c|c|c|}
\hline Authors & $\begin{array}{l}\text { Brewerton } \\
\text { et al. } \\
\text { (2017) } \\
\end{array}$ & $\begin{array}{l}\text { Ornstein } \\
\text { et al. } \\
\text { (2017) }\end{array}$ & $\begin{array}{l}\text { Bryson } \\
\text { et al. } \\
\text { (2018) }\end{array}$ & $\begin{array}{l}\text { Gray } \\
\text { et al. } \\
\text { (2018) }\end{array}$ & $\begin{array}{l}\text { Spettigue } \\
\text { et al. } \\
\text { (2018) }\end{array}$ & $\begin{array}{l}\text { Dumont } \\
\text { et al. } \\
\text { (2019) }\end{array}$ & $\begin{array}{l}\text { Makhzoumi } \\
\text { et al. } \\
\text { (2019) }\end{array}$ & $\begin{array}{l}\text { Lock } \\
\text { et al. } \\
\text { (2019a) }\end{array}$ & $\begin{array}{l}\text { Lange } \\
\text { et al. } \\
(2019)\end{array}$ \\
\hline $\begin{array}{l}\text { Case series collected in more than } \\
\text { one center (i.e. multi-center study) }\end{array}$ & 0 & 0 & 0 & 0 & 0 & 0 & 0 & 0 & 0 \\
\hline $\begin{array}{l}\text { Is the hypothesis/aim/objective of } \\
\text { the study clearly described? }\end{array}$ & 1 & 1 & 1 & 1 & 1 & 1 & 1 & 1 & 1 \\
\hline $\begin{array}{l}\text { Are the inclusion and exclusion } \\
\text { criteria clearly reported? }\end{array}$ & 1 & 1 & 1 & 1 & 1 & 1 & 1 & 1 & 1 \\
\hline $\begin{array}{l}\text { Is there a clear definition of the } \\
\text { outcomes reported? }\end{array}$ & 1 & 1 & 1 & 1 & 1 & 1 & 1 & 1 & 1 \\
\hline Were data collected prospectively? & 1 & 0 & 0 & 1 & 1 & 1 & 0 & 1 & 0 \\
\hline $\begin{array}{l}\text { Is there an explicit statement } \\
\text { that patients were collected } \\
\text { consecutively? }\end{array}$ & 0 & 1 & 1 & 1 & 1 & 0 & 1 & 0 & 1 \\
\hline $\begin{array}{l}\text { Are the main findings of the study } \\
\text { clearly described? }\end{array}$ & 1 & 1 & 1 & 1 & 1 & 1 & 1 & 1 & 1 \\
\hline $\begin{array}{l}\text { Are the outcomes stratified (e.g. } \\
\text { by disease stage, abnormal test } \\
\text { results, patients' characteristics)? }\end{array}$ & 0 & 0 & 0 & 0 & 0 & 0 & 0 & 0 & 0 \\
\hline Total Score & 5 & 5 & 5 & 6 & 6 & 5 & 5 & 5 & 5 \\
\hline
\end{tabular}

La NICE guidelines checklist: Yes = 1, No (not reported, not available) = 0; Total Score: $\mathbf{5} .<3$, low quality; 4-6, moderate quality; $\geq 7$, high quality.

Table 2. Quality appraisal for controlled studies

\begin{tabular}{|c|c|c|}
\hline Authors & Sharp et al. (2016) & Lock et al. (2019b) \\
\hline \multicolumn{3}{|l|}{ Selection } \\
\hline Represents cases with independent validation & 1 & 1 \\
\hline Cases are consecutive or obviously representative & 0 & 1 \\
\hline Controls are from community & 1 & 1 \\
\hline Controls have no history of ARFID & 0 & 0 \\
\hline \multicolumn{3}{|l|}{ Comparability } \\
\hline Controls are comparable for the most important factors. & 1 & 1 \\
\hline Control for any additional factor & 0 & 0 \\
\hline \multicolumn{3}{|l|}{ Ascertainment of exposure } \\
\hline Secured record or structured interview where blind to case/control status & 0 & 1 \\
\hline Same method of ascertainment for cases and controls & 1 & 1 \\
\hline Total Score & 4 & 6 \\
\hline
\end{tabular}

Newcastle-Ottawa Scale (NOS). Yes = 1, No (not reported, not available) = 0; Total Score: 5. 0-3, low quality; 4-6, moderate quality; 7-9, high quality. 
Table 3. Studies included in the systematic review

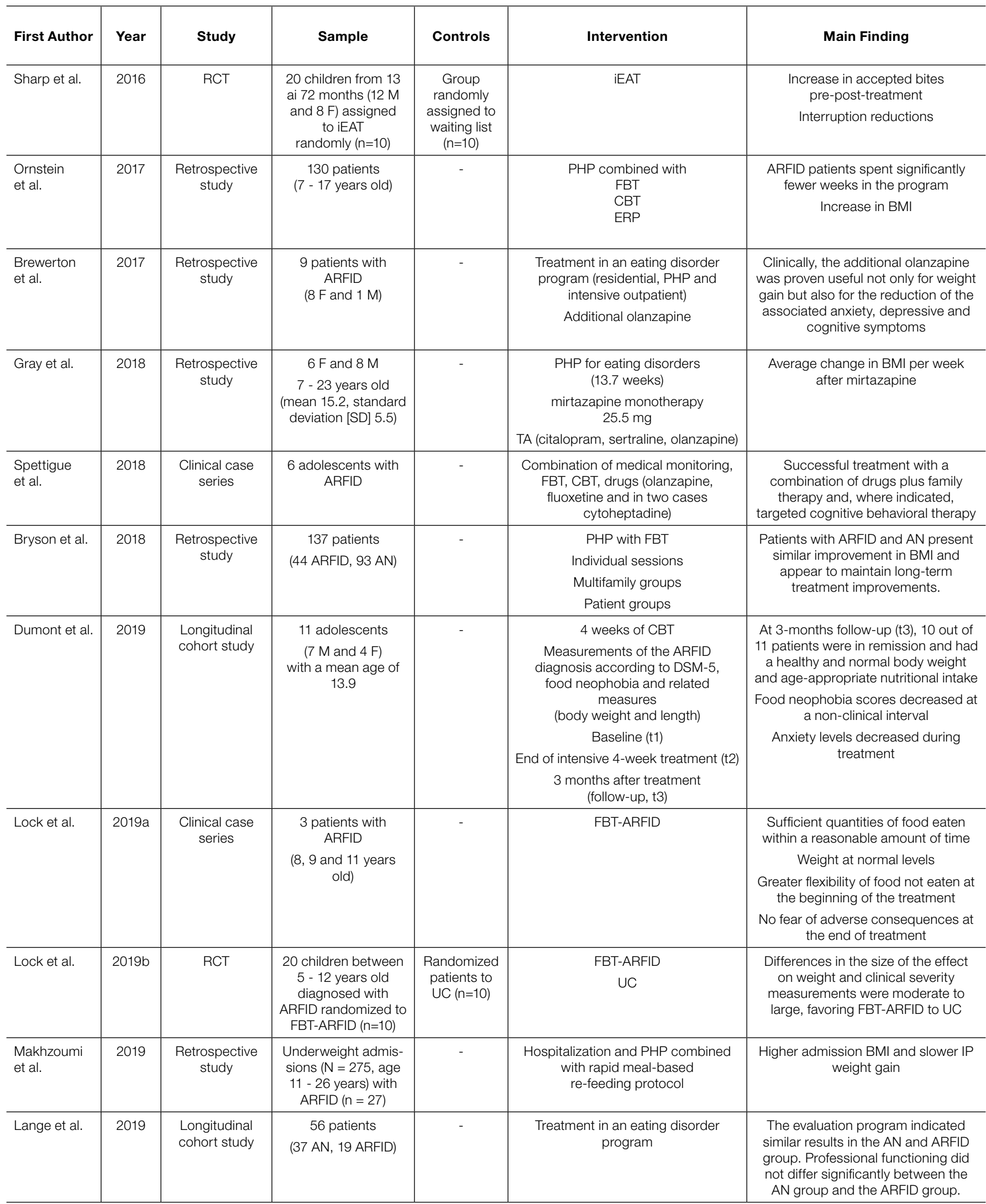

M: male; F: female; AN: anorexia nervosa; CBT: cognitive behavioral therapy; FBT: family-based therapy; UC: usual treatment; RRCT: randomized controlled trial; BMI: body mass index; PHP: partial hospital program; TA: additional therapy; FBT-ARFID: family-based therapy for ARFID; ERP: exposure and response prevention; iEAT: integrated food aversion treatment. 


\section{Data Synthesis}

The studies deemed fit for inclusion were subjected to a narrative review. A narrative review is discursive and seeks to summarize the current state of knowledge in relation to a particular domain by considering a wide variety of sources and reaching conclusions through reason or argument (Popay et al., 2005). In the narrative review, the year of publication of the study, the experimental design of the study (e.g. randomized controlled trial or RCT, retrospective, longitudinal cohort, case series), the sample size, the type of intervention and the main findings of the intervention were described.

\section{Results}

The initial search retrieved 119 papers. After the first screening (title and abstract), 101 papers were excluded for the following reasons: (i) dealt with eating disorders but not ARFID ( $\mathrm{n}=30$ ); (ii) were off-topic ( $\mathrm{n}=27)$; (iii) took into consideration ARFID but not the treatments $(\mathrm{n}=44)$. Of the remaining 18 papers, four descriptive studies and three case reports, with less than two participants, were excluded. Therefore, 11 papers on treatment for ARFID were included in the systematic review and underwent narrative analysis (Figure 1).

\section{Narrative Synthesis}

Sharp and colleagues (2016) conducted a randomized controlled trial (RCT) on a sample of 20 children to study the feasibility and preliminary efficacy of a behavioral feeding intervention based on an active and persistent integrated food aversion treatment (iEAT) for children with chronic food refusal and dependency to enteral feeding or oral nutritional formula supplementation. To be eligible, male ( $\mathrm{n}$ $=12)$ and female $(\mathrm{n}=8)$ participants had to be between 12 months and six years of age, considered appropriate for iEAT and meet the diagnostic criteria for ARFID. Participants in the intervention group $(n=10)$ were engaged in treatment for five consecutive days immediately following baseline. Waiting list children in the control group $(n=10)$ were offered treatment following the 5th day of evaluation. The treatment was provided for 137 meals (97.8\%) out of the 140 scheduled. All final treatment protocols involved operant conditioning procedures (e.g. escape from extinction, differential reinforcement) commonly identified during interventions for serious eating disorders. Six of the nine participants achieved required stability levels in behavior during meals, allowing for a systematic increase in bite volume from a level spoon $\left(2.0 \mathrm{~cm}^{3}\right)$ from meal \#14. Analyzes of the change scores between the study groups were significant in all measures, all favorable to the iEAT group. Children assigned to iEAT showed a significantly greater increa-

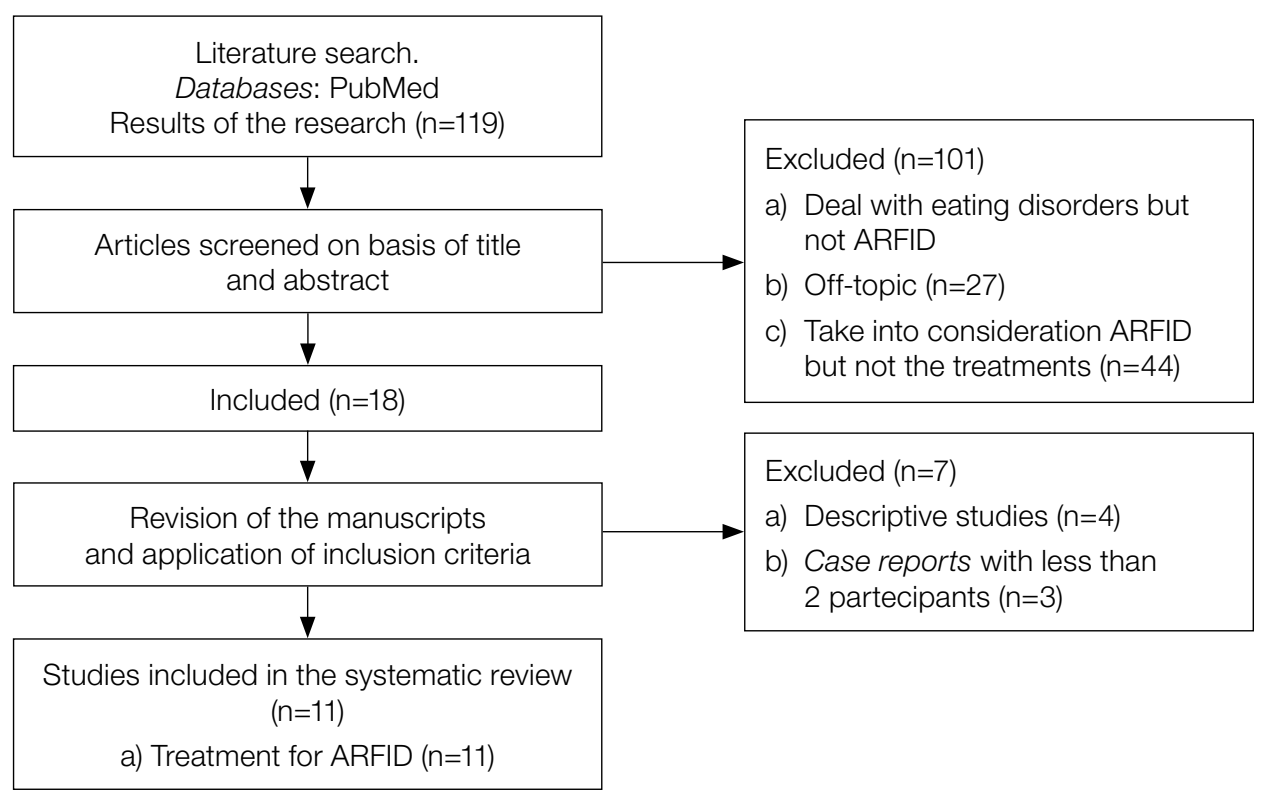

Figure 1. Flow chart summarizing the study selection procedure 
se in pre-post-treatment accepted bites than the waiting list group ( $88.9 \%$ vs $5.6 \%$, respectively) and significantly greater reductions in interruptions (55.6\% vs 9.2\%). Behavioral improvements coincided with a significant increase in the volume of food consumed by children in the iEAT group after treatment (31 grams net in the 10-minute observation). Seven of the nine iEAT participants $(78 \%)$ returned to the clinic for post-treatment follow-up at 36 days (interval 31-60 days). The average accepted bites were $100 \%$ (range 50 - 100) and the interruptions were $13 \%$ (range 0 - 100). Furthermore, a significant increase in the grams consumed at follow-up (median: 71; interquartile range (IQR): 12 - 140) compared to post-intervention (median 34; IQR: 18 - 39; P 1/4 0.031).

Ornstein and colleagues (2017) conducted a retrospective study of 130 patients between the ages of seven and 17 admitted to a partial hospital program (PHP) from 2008 to 2012. The sample was $92.3 \%$ of female sex with a mean age of $13.53+/-2.05$ years with a mean BMI percentile of $88.10 \pm$ 13.74 (range 61.83-156.22). PHP included FBT-derived procedures associated with behavioral, cognitive behavioral, and exposure with response prevention interventions. The intervention was individualized to treat each patient with ARFID and other eating disorders. The response prevention procedure was conceptualized by considering the foods that patients avoided because of fears, worries or disgust. The treatment team worked with participants and their families to identify the foods avoided, explore beliefs about consuming these foods, facilitate their reintroduction during PHP and at home in a generally hierarchical way and discuss the accuracy of predictions related to avoided foods. Almost all participants with ARFID who did not report specific food fears expressed disgust and/or negative beliefs about eating. On average, patients had lost $14.21+/-9.51 \%$ (range 0.00 49.20) of their body weight before starting treatment with a disease duration of $10.25+/-9,87$ months (interval 0.5060.00 ). Although a lower percentage of patients with ARFID $(15.8 \%)$ were transferred to a stationary unit during treatment compared to those with anorexia nervosa (22.7\%), bulimia nervosa $(21.1 \%)$ and other eating disorders with specification or without specification (28.6\%), this difference was not statistically significant, $\chi^{2}(3)=1.43, p=.70$. Patients who were discharged to a more intensive care level lost a significantly greater percentage of their body weight before starting PHP treatment $(19.46 \%$ vs $13.46 \%)$ than patients who completed the treatment (14.21\% vs $6.51 \%), \mathrm{t}(158)=$ $2.62, \mathrm{p}=.01$. These two groups did not differ significantly in terms of age, sex, BMI \% at the time of engagement, disease duration or intake scores in the ChEAT (children's eating attitude test).
Brewerton and D'Agostino (2017) conducted a retrospective study of nine patients with ARFID treated in a program for eating disorders (residential, partial hospital and intensive outpatient) with the administration of additional olanzapine. After admission to the program, patients were treated with a structured behavioral program, which included assisted meals six times a day (three meals and three snacks) adapted for patients with ARFID. In addition, patients received all the other treatment modalities offered to patients with eating disorders, including individual, group and family therapies, nutritional advice and pharmacotherapy. If patients did not respond to the structured program demonstrating an improvement in food consumption and weight gain during the initial phase of treatment (an average weight gain of $1 \mathrm{lb}$ per week), they were offered low-dose olanzapine therapy, which was started at a dose of $0.625 \mathrm{mg}$ before bedtime in seven of the nine cases (age 9-18 years), $1.25 \mathrm{mg}$ in one case (age 17 years) and $2.5 \mathrm{mg}$ in another case (age 18 years and only because the instructions for cutting the pill were not followed by the patient). The dosage was then administered slowly based on clinical response and side effects. Daily eating behavior, weight and psychological responses were monitored on an ongoing basis. The initial and final mean doses of olanzapine were $0.9+0.63 \mathrm{mg} /$ day $2.8+1.47$ $\mathrm{mg} /$ day, respectively. There was a statistically significant difference in weight gain compared to post-olanzapine treatment (3.3-7.3 lbs vs 13.1-7.9 lbs [2.92 - $6.62 \mathrm{lbs}$ SI vs 11.88 - $7.17 \mathrm{lbs} \mathrm{SI}$, paired t-test $(\mathrm{p}<0.04, \mathrm{t}=-2.48))$ Clinically, additional olanzapine was useful not only for weight gain but also for the reduction of associated anxiety, depressive and cognitive symptoms.

Gray and colleagues (2018) conducted a retrospective study of 14 consecutive ARFID patients treated with mirtazapine. The six female and eight male participants were aged between seven and 23 years (mean 15.2, SD 5.5) and had a mean baseline BMI of $16.8 \mathrm{~kg} / \mathrm{m}^{2}$ (SD 1.6, range 13.919.1). The mean duration of treatment was 13.7 weeks (SD 5.2 , range $7-25$ ) and the mean BMI was $19.5 \mathrm{~kg} / \mathrm{m}^{2}$ (SD 1.9, range 16.3-22, 3). Patients were treated for an average of 3.4 weeks (SD 2.7) before starting mirtazapine and were treated with mirtazapine for an average of 9.8 weeks (SD 5.1), continuing with the drug except for one patient who stopped in the last week of treatment due to an increase in nighttime feeding. The average dose of mirtazapine was $25.5 \mathrm{mg}$ (SD 17.9, range 7.5-60). The mean change in BMI before starting mirtazapine was $0.10 \mathrm{BMI}$ per week (SD 0.08), while the mean change in BMI per week after Mirtazapine was 0.23 BMI (SD 0.14) with a statistically si- 
gnificant difference (t13 1/4 3.11, $\mathrm{p}<.05)$. The results suggest that mirtazapine could facilitate faster weight gain in patients with ARFID.

Spettigue and colleagues (2018) conducted a study on six patients diagnosed with ARFID who were sequentially treated by a child psychiatrist and adolescent medicine doctor in a hospital eating disorder program that included FBT. Five out of six cases were female and the mean age of the patients at the evaluation was 12.9 years $(\mathrm{SD}=1.13)$. On average, the target weight percentage of patient treatment was $80.5 \%$ at the initial assessment $(\mathrm{SD}=8.56)$ and $81.9 \%$ $(\mathrm{SD}=7.08)$ at the beginning of FBT. Cases 1,2 and 3 were admitted to a specialized inpatient unit due to medical instability or lack of response to outpatient treatment, and all six cases had severe levels of comorbid anxiety. All patients were treated using a combination of medical monitoring, FBT, drug therapy (including olanzapine, fluoxetine and in two cases cyproheptadine) and CBT. By the end of the treatment, all six patients had reached their target weight.

Bryson and colleagues (2018) conducted a retrospective study of 137 patients in which patients with ARFID ( $\mathrm{n}=$ $44)$ and anorexia nervosa $(\mathrm{n}=93)$ were compared more than 12 months after being discharged from PHP which included FBT, individual sessions, multi-family, and patient groups. The treatment was administered by a multidisciplinary team with an approach that combined family therapy procedures with cognitive-behavioral interventions, including cognitive restructuring, meal planning, behavior modification techniques and flexible exposure to foods that patients avoided due to fear, disgust, and/ or worries of adverse outcomes. Patients were also provided with psychotropic drugs at the discretion of the team psychiatrist. All participants showed a significant increase in BMI percentiles from entry to discharge $[\mathrm{F}(1,57)=$ 198.80, $\mathrm{p}<.001, \mathrm{r}=.88$ ]. Participants with ARFID and anorexia nervosa maintained their BMI percentile at follow-up, which was slightly higher than their BMI percentile at discharge $[\mathrm{F}(1,57)=1.86, \mathrm{p}=.18]$. The anorexia nervosa group achieved a greater increase in BMI percentile from entry to discharge than the ARFID group [F (1, $57)=8.77, \mathrm{p}<.01, \mathrm{r}=.37$ ], but the two diagnostic groups showed no significantly different changes from discharge to follow-up $[\mathrm{F}(1,57)=0.24, \mathrm{p}=.63]$.

Dumont and colleagues (2019) conducted a longitudinal cohort study, on 11 patients with ARFID, using a specific four-week form of exposure-based CBT. Measurements of the DSM-5 ARFID diagnosis, food neophobia and related measures such as body weight and height, were taken before baseline ( $\mathrm{t} 1)$, at the end of the intensive four-week treatment (t2) and at three months follow-up (t3). Participants completed a test that assessed food selectivity and a one-week food diary at $\mathrm{t} 1$ and $\mathrm{t} 3$. In addition, continuous measurements of beliefs, anxiety and food acceptance were taken during the 4-week intensive treatment. Patients received 75 to participate in the follow-up measurement. At the end of treatment ( $\mathrm{t} 2)$, six patients (55\%) no longer met any of the ARFID criteria. Average anxiety levels decreased from baseline to week 1-4 of intervention in seven (64\%) patients. In $91 \%$ of patients $(n=10)$, food acceptance had increased while selectivity had decreased at three months follow-up (t3), compared to baseline measurements (t1). The nine patients (82\%) who participated in the follow-up showed an increase in the number of foods accepted and consumed during and after the intervention.

Lock and colleagues (2019a) conducted a study on three case reports to illustrate how FBT can be used to treat preadolescents with ARFID. These case reports showed that FBT can be used in three different clinical presentations of ARFID: (i) poor appetite and lack of interest; (ii) sensitivity to sensory characteristics; and (iii) fear of adverse consequences - all without the presence of concerns about weight and body shape. The authors, commenting on their treatment experience, reported that common challenges in this group of patients include: (i) managing medical emergency; (ii) demanding long-term behavioral accommodation; (iii) lack of alignment and fatigue of parents and (iv) psychiatric problems in co-morbidity in patients. According to the authors, FBT can be adapted to children with ARFID using the basic principles of the intervention.

Lock and colleagues (2019b) conducted an RCT on 20 patients with ARFID randomized to FBT-ARFID $(\mathrm{n}=10)$ or usual treatment (UC). Parents who received FBT-ARFID reported an average score of $8.00(\mathrm{SD}=1.66)$ on the suitability of FBT-ARFID for their child's condition and 6.56 $(\mathrm{SD}=2.35)$ on the expectation of success of treatment. The drop-out rate was $21 \%$ for the whole sample. The difference in the effect size (ES) in the scores of the changes in the $\%$ of expected body weight (EBW) was Cohen's $d=-0.87$ in favor of FBT-ARFID. Similarly, examining the scores of the variations between the groups on the variation of the severity levels of the Pica, ARFID, and Rumination Disorder Interview (PARDI) (Bryant-Waugh et al., 2019) between the groups, an ES of 0.83 emerged, favoring those who received FBT-ARFID. These data provide some support for the hypothesis that FBT-ARFID is a potential more effective treatment for ARFID than UC. Parental self-efficacy changes were greater in FBT-ARFID than in UC $(\mathrm{d}=-1.49)$. Furthermore, the change in parental self-efficacy was hi- 
ghly correlated with improvements in EBW\% with an overall Spearman Rho of 0.46 at EOT. For the FBT-ARFID group, Spearman's Rho was 0.83 , while for the UC, -0.16 at the end of the UC. Taken together, these more frequent changes in parental self-efficacy in relation to feeding are a possible mechanism of action of FBT-ARFID.

Makhzoumi and colleagues (2019) conducted a retrospective study on hospitalizations of consecutive underweight patients ( $\mathrm{N}=275$, age 11-26 years) treated with a combination of interventions (CBT, FBT, DBT, UC) with ARFID $(\mathrm{n}=27)$, compared with anorexia nervosa $(\mathrm{n}=248)$ on clinical characteristics, the reason for discharge and weight recovery variables in a PHP. The mean age was 18.97 years $(\mathrm{SD}=3.60)$ with $42 \%$ under 18 years old. $90 \%$ of the participants had a diagnosis of anorexia nervosa (44\% restrictive type and $46 \%$ with binge eating/purging behaviors) and $10 \%$ had an ARFID diagnosis. Over three-quarters of the sample were included in the ARFID-aversive subtype with $80 \%$ avoiding gastrointestinal symptoms to restrict food. This was the most common observed reason for the restriction. Nearly a third of the sample $(n=8)$ had a childhood history of growth failure and a history of malnutrition, including all individuals who had ARFID-limited intake as their predominant subtype $(n=5)$. Five patients had a primary fear of vomiting or suffocation in the absence of evidence of the associated symptom. Almost a third of the sample was a mixed ARFID subtype ( $\mathrm{n}=2$ adverse and limited intake type, $\mathrm{n}=1$ adverse and limited variety type, $\mathrm{n}=1$ limited intake and limited variety type, and $\mathrm{n}=$ 4 aversive, limited intake, and limited variety type). About half of ARFID patients reported non-induced vomiting at least once a week in the month preceding admission. Common gastrointestinal diagnoses and symptoms included gastroesophageal reflux disease (GERD) (29\%), gastroparesis (24\%), irritable bowel syndrome (9\%), constipation (19\%), abdominal pain (52\%) and nausea (38\%) with food consumption. Among those who reported gastrointestinal problems, $63 \%$ reported having difficulties in the upper gastrointestinal tract (e.g. vomiting or GERD) and over $80 \%$ reported lower gastrointestinal pain (e.g. chronic constipation, diarrhea or abdominal pain). ARFID patients were more likely to be male. No group differences were observed regarding age at the time of admission. During the course of treatment and in clinical outcomes, the ARFID group had a slower weekly weight gain during treatment than the group with anorexia nervosa, a smaller percentage attended PHP (48\%) than those with anorexia nervosa (74\%) and spent fewer days in PHP. Patients with ARFID were equally likely to be discharged for clinical improvement compared to those with anorexia nervosa and both groups had a similar BMI at the end of the program ( $\mathrm{p}>0.05)$.

Finally, Lange and colleagues (2019) conducted a longitudinal cohort study to compare the results of an as usual treatment between childhood-onset anorexia nervosa and ARFID, diagnosed retrospectively, in regards to psychiatric diagnoses, social and professional functioning. in a sample of 56 patients with ARFID $(n=19)$ compared with patients with anorexia nervosa $(n=37)$. The mean age at the start of treatment was 11 years (range 6.8-12.9) and follow-up was carried out in the period between 2010 and 2013, with an average time of 15.9 years (range 7.2-29.3). At follow-up, weight and height were measured. Two interviews were conducted: a semi-structured interview developed for follow-up and a structured clinical interview for the diagnosis of mental disorders (Structured Clinical Interview for DSM-IV Axis I Disorders - SCID-I). At the start of treatment, 37 cases were diagnosed with anorexia nervosa and 19 with ARFID. At follow-up, the mean BMI for the anorexia nervosa group was $21.5 \mathrm{~kg} / \mathrm{m}^{2}$ (range 17.4-28.0; SD 2.61) and for the ARFID group $21.9 \mathrm{~kg} / \mathrm{m}^{2}$ (range 16.5- 29.9; DS 3.33). The mean age, at follow-up, of the anorexia nervosa group was 28.4 years (range 18.9-42.2; SD 6.58), while the mean age of the ARFID group was 25.5 years (range 19.4- 40.7; DS 5.35). In the anorexia nervosa group, at follow-up, 21.6\% $(\mathrm{n}=8)$ had an on-going eating disorder, $24.3 \%(\mathrm{n}=9)$ had another psychiatric diagnosis (but not an eating disorder) and $54.1 \%(\mathrm{n}=20)$ had no psychiatric diagnosis. In the ARFID group, at follow-up, 26.3\% ( $n=5)$ had an on-going eating disorder, $26.3 \%(\mathrm{n}=5)$ had another psychiatric diagnosis and $47.4 \%(n=9)$ had no psychiatric diagnosis. In the group with anorexia nervosa, the professional level was $95 \%$ and in the ARFID group $84 \%$. For the subgroup without a psychiatric diagnosis at follow-up, both the anorexia nervosa group and the ARFID group showed $100 \%$ occupational levels. For the subgroup with a current eating disorder, the group with anorexia nervosa had a professional level of $87 \%$ while the ARFID group of $80 \%$. Finally, in the subgroup who had another psychiatric diagnosis at follow-up but who did not have a current eating disorder, the group with anorexia nervosa had a professional level of $89 \%$ while the ARFID group of $60 \%$.

\section{Discussion}

This review aimed to provide reference data on the effects of ARFID treatment. The systematic narrative review included 11 studies including two RCTs, two longitudinal 
cohorts, five retrospectives, and two clinical case studies. Both the controlled and non-controlled studies were judged to be of moderate quality (Tables 1 and 2). In addition, both controlled studies included a small number of participants $(\mathrm{N}=20)$ under the age of 13 and had little statistical power. Only two retrospective studies also included young adults. Finally, most of the studies assessed weight and BMI as the outcome of the treatment. Only a few studies evaluated interruption of treatment, the intake of the amount of food, food flexibility, fear of adverse consequences, interpersonal functioning and symptoms of anxiety as outcome measures.

\section{Summary of Evidence and Limitations}

The systematic review produced three main findings. The first finding with strong evidence is that a significant weight gain occurred at the end of treatment in all participants with ARFID in all the examined studies. The available data do not allow, however, to draw significant conclusions on the difference between the various treatments in this measure of outcome. The only RCT that compared two active treatments (FBT-ARFID versus UC), although a higher effect size was reported on weight and clinical severity measures in favor of FBT-ARFID compared to UC, does not have sufficient statistical power to draw conclusions (Lock, Sadeh-Sharvit, et al., 2019).

The second result with evidence that requires further confirmation, because the outcomes were evaluated only in four studies (Bryson et al., 2018; Dumont et al., 2019; Lange et al., 2019; Sharp et al., 2016), is that weight recovery is maintained at follow-up in a fair percentage of participants and treatments tend to lead to an improvement in eating behavior and flexibility in food intake associated with a reduction in the symptoms of anxiety and depression and a decrease in the fear of adverse consequences. It should be noted, however, that two studies have a follow-up of only 36 days (Sharp et al., 2016) and three months (Dumont et al., 2019), one of 12 months (Bryson et al., 2018) and that the only study with a long-term follow-up showed that after about 15 years from the end of treatment only $47.4 \%$ had no psychiatric diagnosis and that $16 \%$ did not carry out any work activity (Lange et al., 2019).

The third result is that there is weak or missing evidence regarding the treatment of ARFID in adults and in the difference in outcome in the three ARFID profiles described by the DSM-5 (American Psychiatric Association, 2013), i.e. the avoidance and restriction of food for the lack of interest, for the sensory characteristics and for the fear of adverse consequences, and in non-underweight subjects.
The main limitation of the study is that a narrative review cannot provide a quantitative synthesis of the literature that a metanalysis can provide. However, a metanalysis could not be performed because of the different outcome measures used in the studies that were included in this review.

\section{Implications for Future Research}

The current diagnosis of ARFID presents several problems in its definition and individualization, so much so that it has been defined an "umbrella" diagnosis (Zucker, 2016). The first problem concerns the lack of any indication with respect to the psychopathology underlying the behaviors reported by the diagnosis. The second has to do with the total lack of insight into the motivation for the weight loss, in case it occurs. The third, the diagnosis of ARFID is satisfied even when the current difficulties related to nutrition arise in the context of an already identified problem (e.g. selective eating in autism) (Zucker, 2016). Furthermore, a problem that makes it difficult to diagnose ARFID in underweight adolescent and adult subjects, concerns its overlap with anorexia nervosa, because the only real difference between patients with anorexia nervosa and patients with ARFID relates to the perception of seriousness of the underweight present in the latter, but not in the former, while many features overlap, given that for some patients with anorexia nervosa, in addition to the fear of gaining weight, weight loss can also be motivated by the need for control or visceral hypersensitivity (Zucker, 2016).

The difficulty in the diagnostic classification and identification of the specific psychopathology of ARFID makes it difficult to direct the treatment, which to date, based on what emerges from this review, is still not well studied and has many limitations (Brigham, Manzo, Eddy, \& Thomas, 2018; Talbot \& Thornton, 2016). In particular, based on the current knowledge, most of the clinical research continues to be performed in pediatric subjects, in which the chosen intervention remains hospital or PHP or outpatient, using FBT or behavioral exposure techniques, mainly aimed at increasing the volume, the variety of nutrition and, if the patient is underweight, also body weight (Thomas et al., 2017). On the contrary, no controlled trials are available to date in adolescent or adult patients and only case reports and controlled studies with low statistical power, low number of participants and short follow-up have weakly underlined the potential of FBT-ARFID, a treatment derived from FBT of anorexia nervosa (Lock \& Le Grange, 2013; Lock et al., 2010), of behavioral strategies and procedures (Bryant-Waugh, 2013; King, Urbach, \& Stewart, 
2015) and of eclectic forms of treatments in different care settings (outpatient, PHP, hospitalization).

Some authors believe that the specialized treatment models established for eating disorders with restrictions such as anorexia nervosa could potentially be effective in ARFID as well, but the longitudinal studies that used these forms of treatment are not available.

Some North American clinicians have recently proposed a cognitive behavioral therapeutic intervention for ARFID (CBT-AR) based on a three-dimensional model of the neurobiology of ARFID (Thomas et al., 2017; Thomas, Wons, \& Eddy, 2018). The treatment, which has not yet been evaluated by accurate clinical studies and for this reason has not been included in this systematic review, hypothesizes a biological predisposition that, favoring negative emotions or predictions regarding the adverse consequences of eating, could lead to a restriction which in turn would favor nutritional impairment and food intake avoidance. These last two characteristics in turn would maintain the restriction of nutrition through the increase in digestive difficulties related to nutritional impairment and the impossibility of experiencing different / positive food experiences due to social avoidance, thus triggering a vicious circle. However, this efficacy of this therapeutic model has not been yet assessed in large clinical studies and it has not been included in this systematic review.

Future research must accurately characterize the psychopathology of ARFID which, in the current state of the DSM-5 classification, appears very heterogeneous and of doubtful clinical utility in directing the treatment. Once the psychopathology of ARFID is better clarified, it will be possible to evaluate more accurately which mechanisms contribute to maintaining it and this may lead to the development of specific strategies and procedures to deal with it which must then be evaluated in accurate cohort and randomized clinical trials. In addition, it is necessary to evaluate the effects of the treatments in adolescents and adults and in the different profiles of ARFID.

\section{References}

American Psychiatric Association. (2006). Diagnostic and statistical manual of mental disorders, (4th ed.). Washinton, DC.

American Psychiatric Association. (2013). Diagnostic and Statistical Manual of Mental Disorders (5th ed.). Washington, DC.

Brewerton, T. D., \& D'Agostino, M. (2017). Adjunctive Use of Olanzapine in the Treatment of Avoidant Restrictive Food Intake Disorder in Children and Adolescents in an Eating Disorders Program. J Child Adolesc Psychopharmacol, 27(10), 920-922. doi:10.1089/cap.2017.0133
Brigham, K. S., Manzo, L. D., Eddy, K. T., \& Thomas, J. J. (2018). Evaluation and Treatment of Avoidant/Restrictive Food Intake Disorder (ARFID) in Adolescents. Current Pediatrics Reports, 6(2), 107-113. doi:10.1007/s40124-018-0162-y

Bryant-Waugh, R. (2013). Avoidant restrictive food intake disorder: an illustrative case example. Int J Eat Disord, 46(5), 420-423. doi:10.1002/eat.22093

Bryant-Waugh, R., Micali, N., Cooke, L., Lawson, E. A., Eddy, K. T., \& Thomas, J. J. (2019). Development of the Pica, ARFID, and Rumination Disorder Interview, a multi-informant, semi-structured interview of feeding disorders across the lifespan: A pilot study for ages 10-22. Int J Eat Disord, 52(4), 378-387. doi:10.1002/eat.22958

Bryson, A. E., Scipioni, A. M., Essayli, J. H., Mahoney, J. R., \& Ornstein, R. M. (2018). Outcomes of low-weight patients with avoidant/restrictive food intake disorder and anorexia nervosa at long-term follow-up after treatment in a partial hospitalization program for eating disorders. Int $J$ Eat Disord, 51(5), 470-474. doi:10.1002/eat.22853

Dumont, E., Jansen, A., Kroes, D., de Haan, E., \& Mulkens, S. (2019). A new cognitive behavior therapy for adolescents with avoidant/restrictive food intake disorder in a day treatment setting: A clinical case series. Int J Eat Disord, 52(4), 447-458. doi:10.1002/eat.23053

Gray, E., Chen, T., Menzel, J., Schwartz, T., \& Kaye, W. H. (2018). Mirtazapine and Weight Gain in Avoidant and Restrictive Food Intake Disorder. J Am Acad Child Adolesc Psychiatry, 57(4), 288-289. doi:10.1016/j.jaac.2018.01.011

King, L. A., Urbach, J. R., \& Stewart, K. E. (2015). Illness anxiety and avoidant/restrictive food intake disorder: Cognitivebehavioral conceptualization and treatment. Eat Behav, 19, 106-109. doi:10.1016/j.eatbeh.2015.05.010

Lange, C. R. A., Ekedahl Fjertorp, H., Holmer, R., Wijk, E., \& Wallin, U. (2019). Long-term follow-up study of lowweight avoidant restrictive food intake disorder compared with childhood-onset anorexia nervosa: Psychiatric and occupational outcome in 56 patients. Int J Eat Disord, 52(4), 435-438. doi:10.1002/eat.23038

Liberati, A., Altman, D. G., Tetzlaff, J., Mulrow, C., Gotzsche, P. C., Ioannidis, J. P., . . Moher, D. (2009). The PRISMA statement for reporting systematic reviews and metaanalyses of studies that evaluate health care interventions: explanation and elaboration. J Clin Epidemiol, 62(10), e1-34. doi:10.1016/j.jclinepi.2009.06.006

Lock, J., \& Le Grange, D. (2013). Treatment manual for anorexia nervosa: A family-based approach (2nd ed.). New York: Guilford Press.

Lock, J., Le Grange, D., Agras, W. S., Moye, A., Bryson, S. W., \& Jo, B. (2010). Randomized clinical trial comparing family-based treatment with adolescent-focused individual therapy for adolescents with anorexia nervosa. Archives of General Psychiatry, 67(10), 1025-1032. doi:10.1001/ archgenpsychiatry.2010.128

Lock, J., Robinson, A., Sadeh-Sharvit, S., Rosania, K., Osipov, L., Kirz, N., . . Utzinger, L. (2019). Applying family-based treatment (FBT) to three clinical presentations of avoidant/ restrictive food intake disorder: Similarities and differences from FBT for anorexia nervosa. Int J Eat Disord, 52(4), 439446. doi:10.1002/eat.22994 
Lock, J., Sadeh-Sharvit, S., \& L'Insalata, A. (2019). Feasibility of conducting a randomized clinical trial using family-based treatment for avoidant/restrictive food intake disorder. Int $J$ Eat Disord, 52 (6), 746-751. doi:10.1002/eat.23077

Makhzoumi, S. H., Schreyer, C. C., Hansen, J. L., Laddaran, L. A., Redgrave, G. W., \& Guarda, A. S. (2019). Hospital course of underweight youth with ARFID treated with a meal-based behavioral protocol in an inpatient-partial hospitalization program for eating disorders. Int J Eat Disord, 52(4), 428434. doi:10.1002/eat.23049

National Institute for Health and Clinical Excellence. (n.d.). Clinical Guidelines, Appendix 4 Quality of Case Series Form. In.

Norris, M. L., Spettigue, W., Hammond, N. G., Katzman, D. K., Zucker, N., Yelle, K., ... Obeid, N. (2018). Building evidence for the use of descriptive subtypes in youth with avoidant restrictive food intake disorder. Int J Eat Disord, 51(2), 170173. doi:10.1002/eat.22814

Norris, M. L., Spettigue, W. J., \& Katzman, D. K. (2016). Update on eating disorders: current perspectives on avoidant/restrictive food intake disorder in children and youth. Neuropsychiatr Dis Treat, 12, 213-218. doi:10.2147/NDT.S82538

Ornstein, R. M., Essayli, J. H., Nicely, T. A., Masciulli, E., \& Lane-Loney, S. (2017). Treatment of avoidant/restrictive food intake disorder in a cohort of young patients in a partial hospitalization program for eating disorders. Int $J$ Eat Disord, 50(9), 1067-1074. doi:10.1002/eat.22737

Popay, J., Roberts, H., Sowden, A., Petticrew, M., Britten, N., Arai, L., . . D Duffy, S. (2005). Developing guidance on the conduct of narrative synthesis in systematic reviews. $J$. Epidemiol. Community Health.

Richardson, W. S., Wilson, M. C., Nishikawa, J., \& Hayward, R. S. (1995). The well-built clinical question: a key to evidencebased decisions. ACP J Club, 123(3), A12-13. Retrieved from https://www.ncbi.nlm.nih.gov/pubmed/7582737
Sharp, W. G., Stubbs, K. H., Adams, H., Wells, B. M., Lesack, R. S., Criado, K. K., . . Scahill, L. D. (2016). Intensive, Manualbased Intervention for Pediatric Feeding Disorders: Results From a Randomized Pilot Trial. J Pediatr Gastroenterol Nutr, 62(4), 658-663. doi:10.1097/MPG.0000000000001043

Spettigue, W., Norris, M. L., Santos, A., \& Obeid, N. (2018). Treatment of children and adolescents with avoidant/ restrictive food intake disorder: a case series examining the feasibility of family therapy and adjunctive treatments. J Eat Disord, 6, 20. doi:10.1186/s40337-018-0205-3

Stang, A. (2010). Critical evaluation of the Newcastle-Ottawa scale for the assessment of the quality of nonrandomized studies in meta-analyses. Eur J Epidemiol, 25(9), 603-605. doi:10.1007/s10654-010-9491-Z

Talbot, A., ., \& Thornton, C. A. (2016). Treatment of ARFID. In T. Wade (Ed.), Encyclopedia of Feeding and Eating Disorders: Springer Science+Business Media Singapore.

Thomas, J. J., Lawson, E. A., Micali, N., Misra, M., Deckersbach, T., \& Eddy, K. T. (2017). Avoidant/Restrictive Food Intake Disorder: a Three-Dimensional Model of Neurobiology with Implications for Etiology and Treatment. Current Psychiatric Report, 19(8), 54. doi:10.1007/s11920-017-0795-5

Thomas, J. J., Wons, O. B., \& Eddy, K. T. (2018). Cognitivebehavioral treatment of avoidant/restrictive food intake disorder. Curr Opin Psychiatry, 31(6), 425-430. doi:10.1097/ YCO.0000000000000454

Zucker, N. (2016). Avoidant/Restrictive Food Intake Disorder (ARFID). In Encyclopedia of Feeding and Eating Disorders (pp. 1-4). 